\title{
The relationships between atomic charges and magnetic response properties reflects conductivity of BN nanotubes
}

\author{
Jamshid Najafpour $^{1}$, Farrokh Roya Nikmaram ${ }^{1}$, and Fatemeh Firooznasab ${ }^{1}$ \\ ${ }^{1}$ Yadegar-e-Imam Khomeini (RAH) Shahre Rey Branch, Islamic Azad University
}

February 8, 2021

\begin{abstract}
In this work, the potential relation between magnetic response properties (isotropic shielding $\left(\sigma_{\text {iso }}\right)$ and total atomic magnetizabilities, $\mathrm{X}(\Omega)$ ) with QTAIM atomic charges of boron and nitrogen atoms in $(4,4),(5,3)$ and $(7,0)$ single-walled boron nitride nanotubes (SWBNNTs) are investigated at DFT B3LYP/ 6-31G(d) level of theory using periodic boundary condition (PBC) approach. The results show that a liner correlation exists between atomic charges of B and N in $(4,4)$ and $(5,3)$ BNNTs and the isotropic shielding. The results show a solid correlation between chemical shielding and total-atomic magnetizabilities, $\mathrm{X}(\Omega)$ in $(4,4)$ BNNT with the lowest conductivity.
\end{abstract}

